\title{
Model-Based Approach to Recognize the Rectus Abdominis Muscle in CT Images
}

\author{
Naoki KAMIYA ${ }^{\dagger a)}$, Xiangrong ZHOU ${ }^{\dagger \dagger}$, Members, Huayue CHEN $^{\dagger \dagger \dagger}$, Chisako MURAMATSU ${ }^{\dagger \dagger}$, Nonmembers, \\ Takeshi HARA ${ }^{\dagger \dagger}$, and Hiroshi FUJITA ${ }^{\dagger \dagger}$, Members
}

\begin{abstract}
SUMMARY Our purpose in this study is to develop a scheme to segment the rectus abdominis muscle region in X-ray CT images. We propose a new muscle recognition method based on the shape model. In this method, three steps are included in the segmentation process. The first is to generate a shape model for representing the rectus abdominis muscle. The second is to recognize anatomical feature points corresponding to the origin and insertion of the muscle, and the third is to segment the rectus abdominis muscles using the shape model. We generated the shape model from $20 \mathrm{CT}$ cases and tested the model to recognize the muscle in 10 other $\mathrm{CT}$ cases. The average value of the Jaccard similarity coefficient (JSC) between the manually and automatically segmented regions was 0.843 . The results suggest the validity of the model-based segmentation for the rectus abdominis muscle.

key words: abdominal muscles, $X$-ray CT images, shape model, segmentation
\end{abstract}

\section{Introduction}

In the CT images, the skeletal muscles are not paid attention by most of the doctors because their interest lies in the internal organs that are affected by diseases. Therefore, the effective use of image information is expected for the torso CT images, which were imaged not for the diagnosis of muscles but for the other purposes (e.g. diseased organ). Since all torso $\mathrm{CT}$ slices contain muscle regions, we propose an effective use of these images.

To accomplish the automated recognition of skeletal muscles in CT, we have been developing a computeraided diagnosis (CAD) system and have obtained some positive results for the muscle in the chest and abdominal regions [1], [2]. However, a recognition method, which considers the anatomical shape of the muscles, has not yet been realized except for a psoas major muscle region [3] and rectus abdominis muscle [4] in non-contrast CT images. In addition, previous similar studies based on muscles shape or shape model for muscle segmentation have been done in the MR images [5]-[7], in which muscle regions have higher

Manuscript received June 23, 2012.

Manuscript revised October 17, 2012.

${ }^{\dagger}$ The author is with the Department of Information and Computer Engineering, Toyota National College of Technology, Toyota-shi, 471-8525 Japan.

${ }^{\dagger}$ The authors are with the Department of Intelligent Image Information, Division of Regeneration and Advanced Medical Sciences, Graduate School of Medicine, Gifu University, Gifu-shi, 501-1194 Japan.

${ }^{\dagger \dagger}$ The author is with the Department of Anatomy, Graduate School of Medicine, Gifu University, Gifu-shi, 501-1194 Japan.

a)E-mail: n-kamiya@ toyota-ct.ac.jp

DOI: 10.1587/transinf.E96.D.869 contrast.

We propose a method to automatically segment the rectus abdominis muscle in non-contrast torso X-ray CT images [4]. This method is based on generating a shape model for the rectus abdominis muscles and applying it to a recognition process. Finally, we determined the Jaccard similarity coefficient (JSC) to compare the results of manual extraction and this method. In this paper, we evaluated the method using the same 10 training cases used in [4], and different 10 test cases. In the previous paper [4], the test cases were randomly selected from the cases with ages from 20 to 80 . In this study, the test cases are also randomly selected from a group of ages 50 to 70 who are in the same age group as the training dataset.

\section{Model-Based Muscle Segmentation Method}

The model-based approach was effective for segmenting the muscle region with a characteristic shape [3]. The psoas major muscle has the spindle shape which we approximated its 3-D outer shape. In current method, we focus on the cross sectional shape of the rectus abdominis muscle and approximate it by using a quadratic function. Figure 1 shows the flowchart of the proposed method for the muscle recognition based on the model generation.

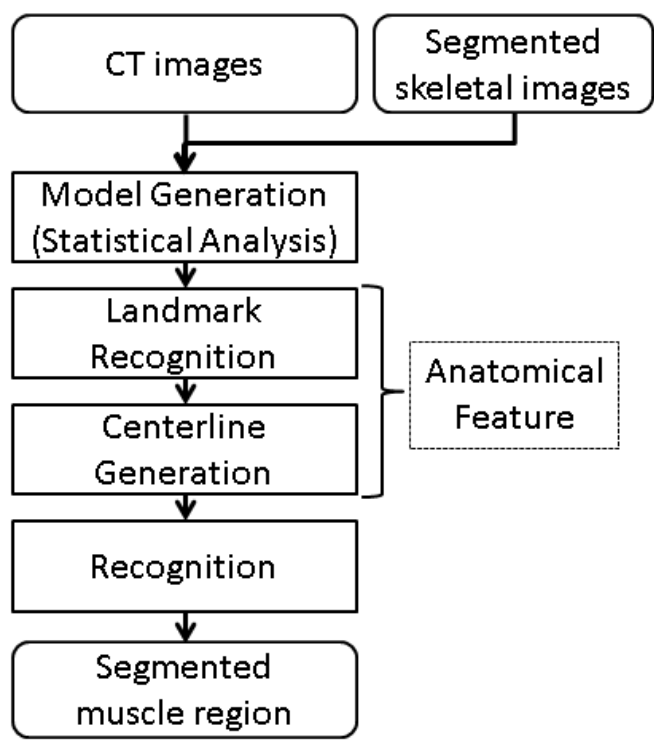

Fig. 1 Processing flow of the muscle recognition. 
The proposed method is based on our advanced scheme. First, the shape model of the rectus abdominis muscle is generated using the training dataset. In the muscle recognition process, the system determines the anatomical feature points as landmarks (LMs) and generates the centerline connecting the LMs for a test case. Finally, the shape model is applied to segment the rectus abdominis muscle region.

\subsection{Statistical Shape Model Generation}

The rectus abdominis muscle has a pathognomonic border shape in its axial transverse section. It has a long flat shape and is located in front of the body [8]. The transverse section of rectus abdominis muscle has an arching shape. In this method, we focused on its cross-sectional shape for model generation. As an initial examination, we considered the shapes of the left and right rectus abdominis muscles the same, because of their symmetry. In addition, the difference in muscular thickness for different transverse sections from its origin to insertion is visually imperceptible. Therefore, the shape model of the rectus abdominis muscle is defined as the following quadratic function that approximates the border shape in the axial transverse section:

$$
\boldsymbol{y}=\alpha \boldsymbol{x}^{2}+8.0
$$

where $\boldsymbol{y}$ represents the arcate counter-shape in the axial transverse section, which is obtained by substituting the distance along the centerline for $\boldsymbol{x}$. For the shape model of rectus abdominis, there are two parameters, $\alpha$ (coefficient) and 8.0 (constant). $\alpha$ is a fitting parameter that is automatically determined for each case in the following recognition process. Conversely, 8.0 is an outer shape parameter that corresponds to the thickness of the rectus abdominis, and is determined beforehand. 8.0 is determined by the average thickness measured manually and assumed by the statistical analysis in twenty training cases.

\subsection{Anatomical Features Recognition}

In the process to recognize the anatomical features, landmarks (LM) and centerlines are determined based on the anatomical positional features. The anatomical positions where the rectus abdominis muscle is connected to skeleton are defined as origin and insertion.

In the LM recognition process, the origin LM is obtained from one point on the crista pubica, and the LMs of the insertion are obtained from a total of four points located on the xiphisternum and on costa V to costa VII. The targeted skeletons are selected from the segmented skeletal image [9], and each LM is determined on the selected skeleton.

In the centerline building process, we automatically connect the LMs that were determined above. The origin and its corresponding insertion are ordinarily connected by the shortest possible straight line. However, the shape of the abdomen differs greatly by individuals. Therefore, the course of the muscle fiber cannot be simply expressed by the straightforward connection of origin and insertion. Therefore, a new additional LM that takes into account for the individual body shape is dynamically acquired on the basis of the LMs that were already set automatically. The additional LM is placed in the middle of the origin and insertion at the cross section. This LM is then moved to the innermost subcutaneous fat within the cross-sectional slice of the rectus abdominis muscle from the anterior to posterior direction. Figure 2 shows the effect of introducing the midpoint. Figures 2 (a) and (b) show the 3D image of the centerlines and the slice image at the mid-point, respectively. The mid-points are placed on the left muscle, while they are intentionally removed so that the centerline was kept straight on the right muscle. The centerlines at the left side are in contact with the muscle and reflecting the individual body shape.

\subsection{Automatic Recognition of the Muscle}

In the recognition process, an appropriate fitting parameter $\alpha$ is obtained from the input image by using the model function in Eq. (1). The $\alpha$ can obviously be determined from Eq. (1) by the coordinate pair of $x$ and $y$. Here, the left and right centerline points in each slice are used for substitution of $(x, y)$. Then, in the model fitting, the area represented by the generated function is assumed to be a mask image, from

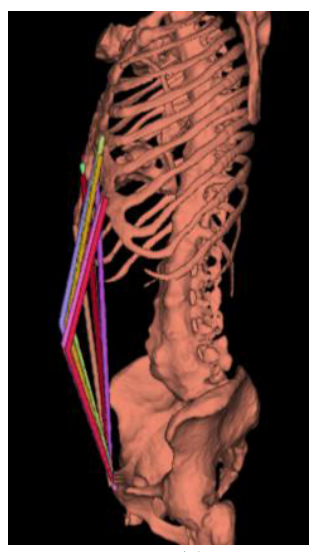

(a)



(b)
Fig. 2 The effect of the mid-point (left side): (a) 3D image of the centerlines, (b) slice image at the mid-point.

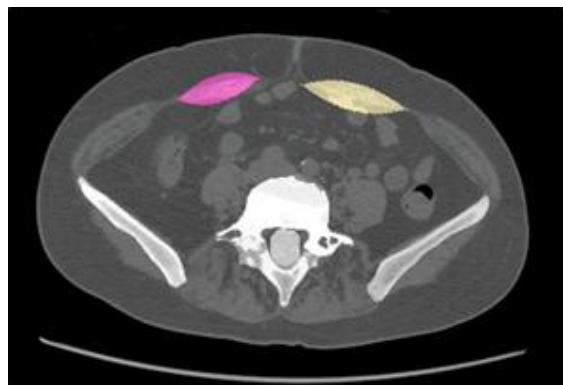

Fig. 3 Shape model on the CT image. 
which the candidate region is obtained. Finally, the muscle region is determined from the peripheral region, if necessary. Figure 3 shows the shape model on the CT image. Each color shows the model for left and right rectus abdominis muscles, respectively.

\section{Results and Discussion}

We applied this scheme to $10 \mathrm{CT}$ cases of non-contrast torso $\mathrm{X}$-ray CT images and evaluated the accuracy of the segmentation results. This study was approved by the Institutional Review Boards. The results were evaluated by the overlap rate between the automatically recognized region and manually extracted region by an author (N.K.) under the guidance of an anatomical specialist. The Jaccard similarity coefficient (JSC) was used to evaluate the proposed method. The JSC is calculated by $(A \cap B) /(A \cup B)$, where $A$ and $B$ are the regions of the gold standard and recognition results, respectively. The test database consisted of CT images of 5 males and 5 females. The method was implemented in a computer with two $2.99 \mathrm{GHz}$ clock CPUs.

We evaluated this method by comparing the recognition results between the previous study [4] and this study with different test datasets which were randomly selected from different age groups. The average JSC value was 0.843 . This result is comparable with that of the previous study (0.841), indicating the muscle recognition using the proposed model has high robustness. Figure 4 shows the recognition results. The green regions are the results extracted with our proposed scheme. The minimum and maximum value of the JSC was 0.831 and 0.892 , respectively. In the proposed technique, the boundary of the muscle was distinguished by using the shape model even if the rectus abdominis muscle touches the area of the intestines. The results indicate the possibility of using the model for automatic recognition of the rectus muscle of abdomen extending to abdominal cavity boundary.

In the proposed method, the anatomical shape could be reproduced by few parameters, and the application of our conventional model-based method [3] can be realized. This indicated a potential for the application of the model-based muscle recognition to other muscles. However, in order to



Fig. 4 Recognition result of the rectus abdominis muscle. verify the validity of this method, it is necessary to apply this method to database with larger variability.

\section{Conclusion}

We proposed a fully automated method based on the shape model to segment rectus abdominis muscle in torso CT images. This method was applied to $10 \mathrm{CT}$ cases. The average values for the JSC was 0.843 . The results were satisfactory in generating the muscle shape model for segmenting rectus abdominis muscle. However, an improvement in accuracy is needed using a large dataset.

\section{Acknowledgments}

The authors would like to thank the other members, Ryujiro Yokoyama, Masayuki Kanematsu and Hiroaki Hoshi in Gifu University for their valuable discussion. This research was funded in part by a grant-in-aid for scientific research on innovative areas, MEXT, Japan, the Naito research grant, and by the ministry of Health, Labour, and Welfare under a grant-in-aid for cancer research, Japan.

\section{References}

[1] X. Zhou, N. Kamiya, T. Hara, H. Fujita, H. Chen, R. Yokoyama, and H. Hoshi, "Automated segmentation and recognition of abdominal wall muscles in X-ray torso CT images and its application in abdominal CAD," Int. J. CARS, 2, Supplement 1, pp.S389-S390, 2007.

[2] N. Kamiya, X. Zhou, H. Chen, T. Hara, H. Fujita, H. Hoshi, R. Yokoyama, and M. Kanematsu, "Automated recognition of structure of abdominal skeletal muscles from X-ray CT images," CARS 2009, S324-5, 2009.

[3] N. Kamiya, X. Zhou, H. Chen, C. Muramatsu, T. Hara, R. Yokoyama, M. Kanematsu, H. Hoshi, and H. Fujita, "Automated segmentation of psoas major muscle in X-ray CT images by use of a shape model: Preliminary study," Radiological Physics and Technology, vol.5, no.1, pp.5-14, 2012.

[4] N. Kamiya, X. Zhou, H. Chen, C. Muramatsu, T. Hara, R. Yokoyama, M. Kanematsu, H. Hoshi, and H. Fujita, "Automated segmentation of recuts abdominis muscle using shape model in X-ray CT images," Proc. IEEE Engineering in Medicine and Biology Society, pp.79937996, 2011.

[5] B. Gilles and N. Magnenat-Thalmann, "Muscloskeletal MRI segmentation using multi-resolution simplex meshes with medial representations," Medical Image Analysis, vol.14, no.3, pp.291-302, 2010.

[6] L. Scheys, D. Loeckx, A. Supaepen, P. Suetens, and I. Johnkers, "Atlas-based non-rigid image registration to automatically define lineof-action muscle models: A validation study," J. Biomechanics, vol.46, no.7, pp.767-772, 2009.

[7] S. Andrews, G. Hamarnehm, A. Yazdanpanah, B. HajGhanbari, and W.D. Reid, "Probabilistic multi-shape segmentation of knee extensor and flexor muscles," MICCAI2011. LNCS, vol.6893, pp.651-658, 2011.

[8] R. Drake, W. Vogl, and A.W.M. Mitchell, Gray's Anatomy for Students, Churchill Livingstone, 2005.

[9] X. Zhou, T. Hayashi, M. Han, H. Chen, T. Hara, H. Fujita, R. Yokoyama, M. Kanematsu, and H. Hoshi, "Automated segmentation and recognition of the bone structure in non-contrast torso CT images using implicit anatomical knowledge," Proc. SPIE, 2009;7259, 72593S. doi:10.1117/12.812945. 